\title{
Prediction of temperature signal of heat exchanger based on ARMA model
}

\author{
Chuan Wang \\ School of Aeronautical Science and Engineering \\ Beihang University \\ Beijing, China \\ E-mail:976615275@qq.com
}

Keywords: ARMA model; heat exchanger; fault temperature signal; prediction

Abstract. With the development of measurement technology, people have accumulated a large amount of data, these data which often with time stamp can be regarded as time series. The basic idea, the process of modeling, and prediction process of fault temperature signal prediction based on ARMA model are presented in this study. Here, we illustrate the utility and challenges of applying $\operatorname{ARMA}(\mathrm{p}, \mathrm{q})$ models, which $\mathrm{p}$ is the dimension of the autoregressive component of the model, and $\mathrm{q}$ is the dimension of the moving average component. We focus on parameter estimation and model selection. The experimental results show that the ARMA model is accurate and feasible in short term fault prediction, results can also provide theoretical basis for the maintenance of the heat exchanger.

\section{Introduction}

Fault prediction is the technology using the current system status as a starting point, combining with known structure features of the object, the parameter, the environmental condition, and historical data, then predicting the system fault analysis and judgment in the future, determining the nature, the category, the degree, the reason and position of the fault, , pointing out the development trend of fault and consequences to eliminate the fault[3]. We hope that with abnormal symptom which is tiny, we can forecast the development of the fault signal[4]. The time series method uses the measured data as a random sequence, according to the measured value which is dependent, then establishing mathematical model to fit time series, It can achieve the effective prediction information accurately [2].

\section{time series model}

For the stationary time series, if at a certain moment the observed value is not only related to the previous observation values, but also has relationship with disturbance before, it is the autoregressive moving average process called the ARMA model. If the observed value associated with the observed value before, it is a AR model. If the observed value disturbance has a relationship with disturbance before only, it is a MA model. the ARMA model is a special case of the AR model and the MA model[1].

MA (q) model [5] :

$$
Y_{t}=\varepsilon_{t}+\theta_{1} \varepsilon_{t-1}+\theta_{2} \varepsilon_{t-2}+\mathrm{L}+\theta_{q} \varepsilon_{t-q}
$$

$\mathrm{AR}(\mathrm{p})$ model:

$$
Y_{t}=\phi_{1} Y_{t-1}+\phi_{2} Y_{t-2}+\mathrm{L}+\phi_{p} Y_{t-p}+\varepsilon_{t}
$$

Given conditions: $1-\phi_{1} z-\phi_{2} z^{2}-\mathrm{L} \phi_{p} z^{p}=0$, the stationary series is the process which the root of the equation falls outside the unit circle.

$\operatorname{ARMA}(\mathrm{p}, \mathrm{q})$ model:

$Y_{t}=\phi_{1} Y_{t-1}+\phi_{2} Y_{t-2}+\mathrm{L}+\phi_{p} Y_{t-p}+\varepsilon_{t}+\theta_{1} \varepsilon_{t-1}+\theta_{2} \varepsilon_{t-2}+\mathrm{L}+\theta_{q} \varepsilon_{t-q}$ 
Given conditions: $1-\phi_{1} z-\phi_{2} z^{2}-\mathrm{L} \phi_{p} z^{p}=0$, the stationary series is the process which the root of the equation falls outside the unit circle. $\left\{\varepsilon_{t}\right\}$ is the white noise sequence.

by introducing the backward shift operator $\mathrm{L}\left(L x_{t}=x_{t-1}\right)$, equation (1), (2), (3) can be written as: $\operatorname{MA}(\mathrm{q})$ :

$$
Y_{t}=\left(1+\sum_{j=1}^{q} \theta_{j} L^{j}\right) \varepsilon_{t}
$$

$\operatorname{AR}(\mathrm{P})$ :

$$
\left(1-\sum_{j=1}^{p} \phi_{j} L^{j}\right) Y_{t}=\varepsilon_{t}
$$

$\operatorname{ARMA}(p, q):$

$$
\left(1-\sum_{j=1}^{p} \phi_{j} L^{j}\right) Y_{t}=\left(1+\sum_{j=1}^{q} \theta_{j} L^{j}\right) \varepsilon_{t}
$$

\section{Modeling steps for ARMA model}

A.The data preprocessing.

In order to avoid false regression problems [6]. we need test the stationarity of the data, if the data is nonstationary, it need to be smoothed. In this paper, using the ADF unit root to test (Augmented Dickey Fuller). For the non-stationary time series, we usually adopt the method of finite difference to smooth it.

B. Determining the order of the model

The order of model which has a great deal of uncertainty has a great influence to the accuracy of the model prediction, so it is very important. In this paper, with the sample autocorrelation (ACF) and partial autocorrelation (PACF) diagram is used to roughly estimate the order of the model, and then, we use the AIC criterion for the order of the model autocorrelation function and partial autocorrelation function with the corresponding relationship with model order number in the following table1:

Table 1.ACF, PACF relationship with model order

\begin{tabular}{|l|l|l|l|}
\hline model & AR(p) & MA(q) & AEMA(p,q) \\
\hline ACF & Tailing & q steps truncation & Tailing \\
\hline PACF & P steps truncation & Tailing & Tailing \\
\hline
\end{tabular}

After roughly determine $\mathrm{p}, \mathrm{q}$, with AIC criterion for final determination:

AIC criteria

$$
\begin{gathered}
A I C(p, q)=\ln \hat{\sigma}_{\varepsilon}^{2}(p, q)+2(p+q) / T \\
\text { where }: \hat{\sigma}_{\varepsilon}^{2}(p, q)=\frac{\sum_{t=1}^{T}\left(y_{t}-\hat{y}_{t}\right)^{2}}{T-p-q}, T \text { is sample size } 。 \\
\operatorname{AIC}(p, q)=\min _{p, q} \operatorname{AIC}(p, q)
\end{gathered}
$$

C.Estimate model parameters

There are so many ways to estimate the model parameter. For example, maximum likelihood estimation method, the least squares estimation method, etc [7]. In this paper, the MATLAB system identification toolbox has an armax function for reference number estimation.

D. Inspection of the model

Whether the model is suitable for the data, it usually need to be tested. in this paper, we use the residual analysis to test whether the residual sequence is white noise[7]. if it is white noise, the model can be used to sequence prediction. In this paper, we use LB statistic test [1] . 


$$
\begin{aligned}
& Q_{L B}(m)=N(N+2) \sum_{k=1}^{m}\left(\frac{\rho_{k}^{2}}{N-k}\right) ; \\
& \text { where : } m=\ln (N), \rho_{k}^{2} \text { is residual autocorrel ation function, } \\
& N \text { is sample size. }
\end{aligned}
$$

It can proved that the $\mathrm{Q}$ approximation is subject to $\chi^{2}(m)$ distribution. after giving an significance level, Checking the site $\chi^{2}(m)$.

if $Q<\chi_{\alpha}^{2}(m)$, then accept the null hypothesis, the sequence is white noise;

if $Q>\chi_{\alpha}^{2}(m)$, thenRefuse to null hypothesis, sequence is white noise.

E. Forecasting for temperature signal

the optimal prediction of sequence has been proved to be its conditional expectation:

$$
\hat{Y}_{t+1 \mid t}=E\left(Y_{t+1} \mid X_{1}, X_{2}, \mathrm{~L}, X_{t}\right)
$$

However, in this paper, we use prediction method is linear minimum variance prediction. Suppose $X_{t}=\left(X_{1}, X_{2}, \mathrm{~L}, X_{t}\right), \quad \alpha=\left(\alpha_{1}, \alpha_{2}, \mathrm{~L}, \quad \alpha_{t}\right)$.predictive variable $\hat{Y}_{t+1 \mid t}$.

$$
\begin{aligned}
& \hat{Y}_{t+1 \mid t}=\alpha^{T} X_{t} \\
& \text { where }: \alpha^{T}=\left[E\left(Y_{t+1} X_{t}^{T}\right)\right] \cdot\left[E\left(X_{t} X_{t}^{T}\right)\right]^{-1} .
\end{aligned}
$$

\section{Experimental results}

The common fault of heat exchanger is incrustation scale. the incrustation scale will jam the plate of channels which leads to greatly reduced thermal efficiency. This paper pays attention to the malfunctions due to scaling in heat exchanger through simulation. The sampling interval of experimental data is 30 seconds. Picking the $4 * 164$ data points contained the import and export of the temperature of the hot and cold road of in the process of scaling. The experimental working medium is air. Using 114 data points front to model, then predicting the result of 50 data points, then the comparing it to the real value which can show accuracy of the prediction, in this article the outlet temperature and inlet temperature of hot and cold are predicted(i.e. forecasting the parameters 25 minutes in advance).

Experimental apparatus as shown in figure:

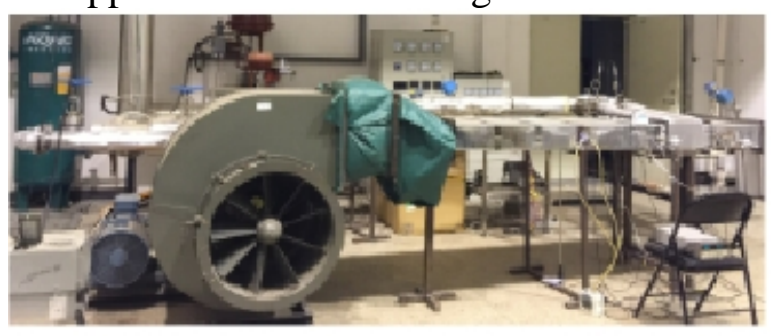

Overall test bench

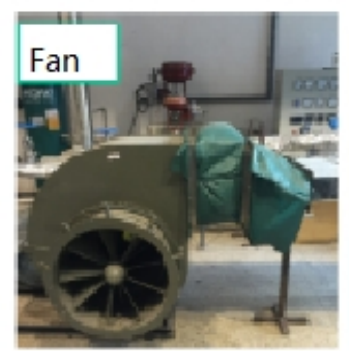

Ram air unit

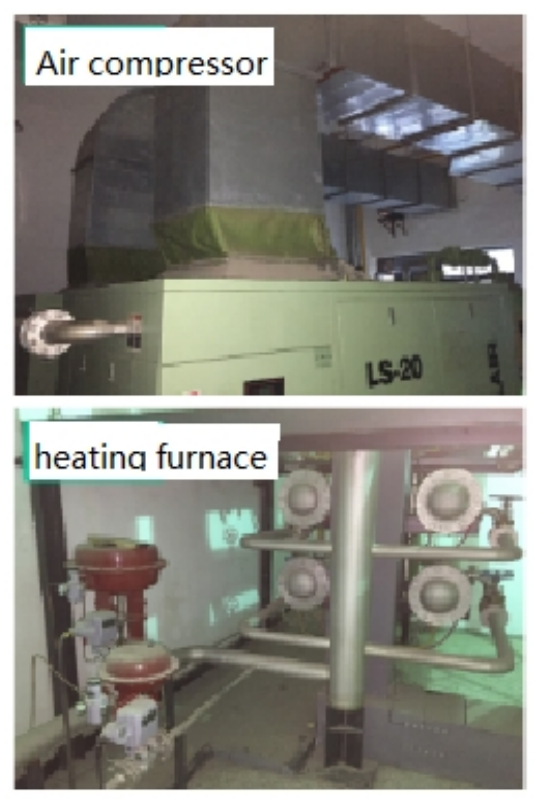

High pressure gas unit

Fig.1 experimental apparatus 
Test data forecasting:
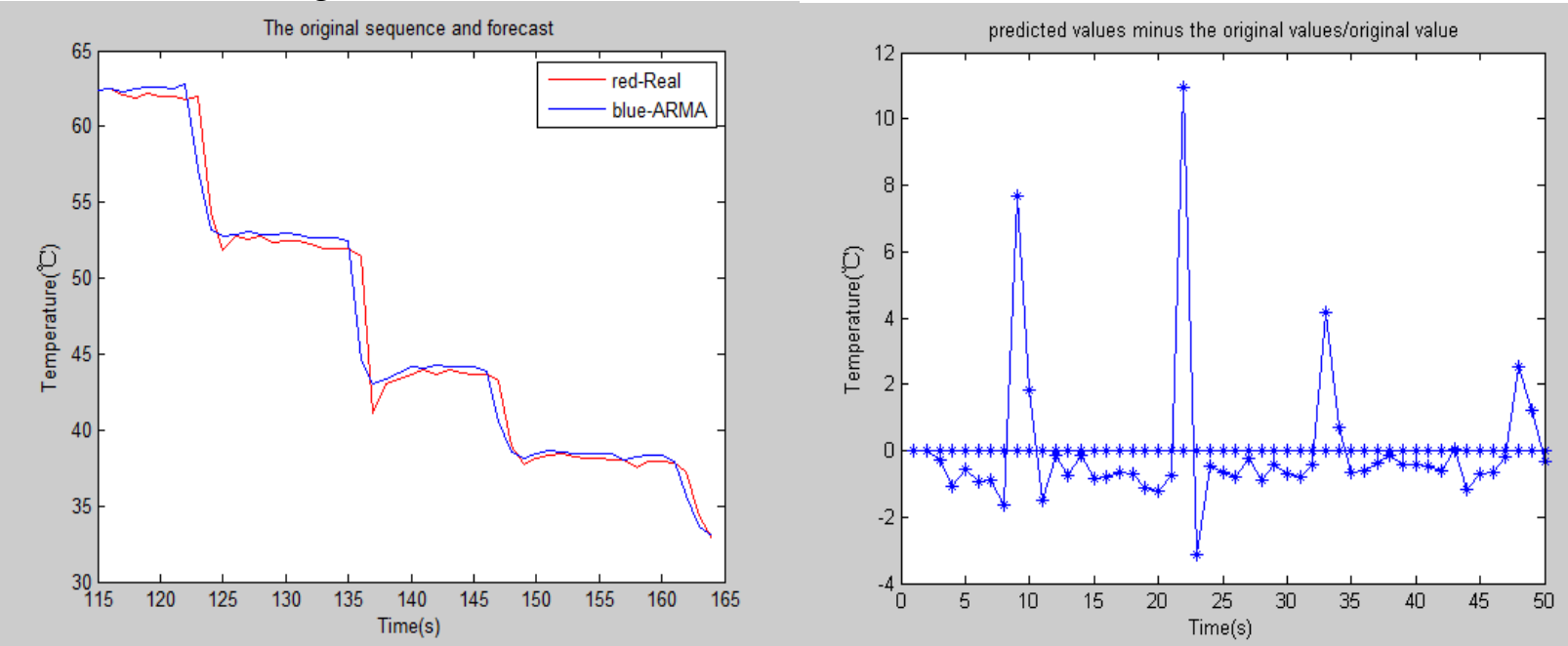

Fig.2 the hot road entrancetemperature prediction

Fig.3 The relative error of hot road entrance temperature
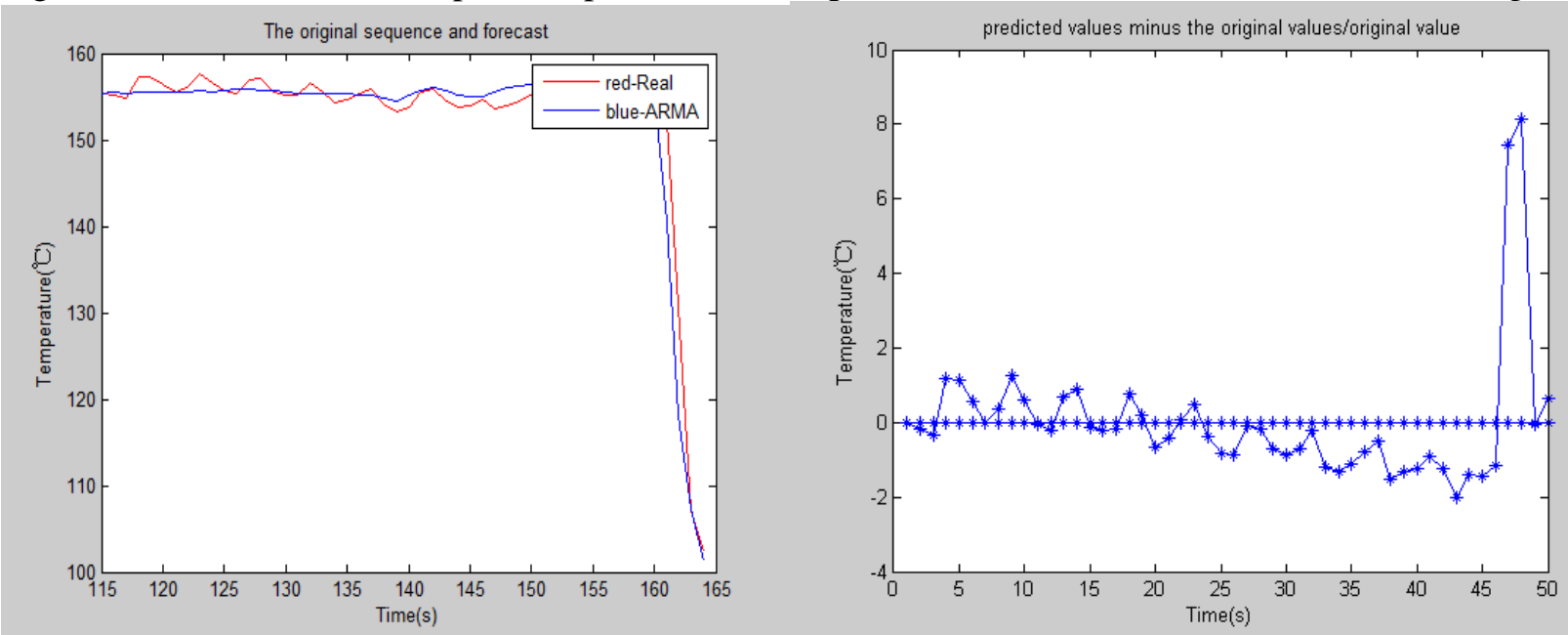

Fig.4The outlet temperature prediction of the hot road Fig.5 The relative error of outlet temperature of hot road
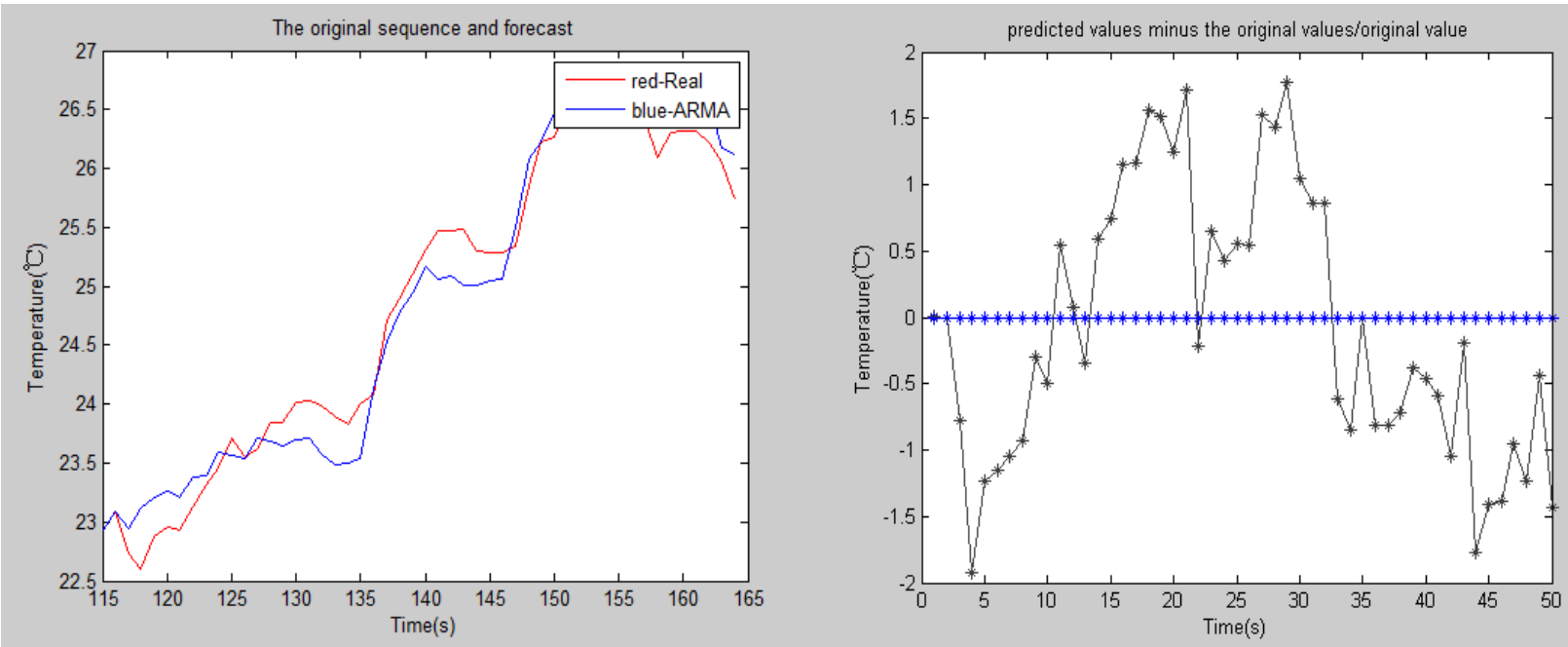

Fig.6 The cold road entrance temperature prediction Fig.7 The relative error of cold road entrance temperature 


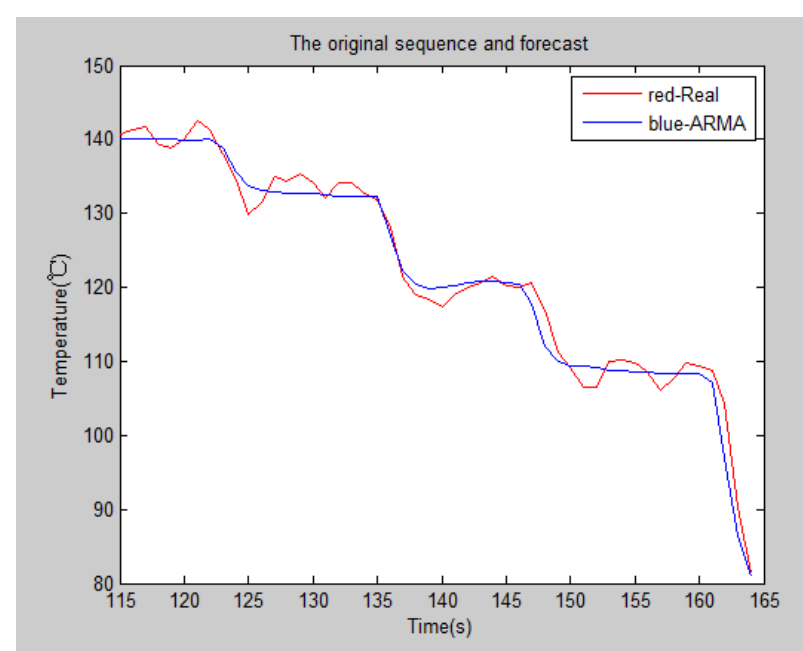

Fig.8 The outlet temperature prediction of the cold road

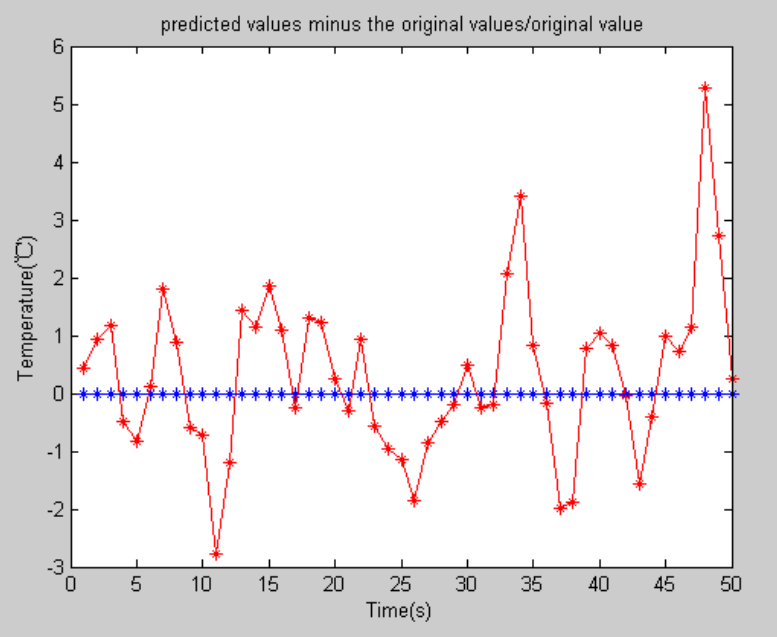

Fig.9 The relative error of outlet temperature of the cold road

In the prediction of the value of Cold road exit temperature back, the error has been relatively larger. it is not suitable for this model. From the data we can see that the ARMA model is suitable for short-term forecasts, accuracy of long-term prediction is not high.

\section{Conclusions}

With the development of modern testing technology, we have a lot of equipment failure data which is a timestamp, in this article, we use time series ARMA model of the heat exchanger in the import and export temperature prediction research, we found that it is suitable for short-term forecasts, which has the very high precision. But it isn't suitable for the long-term forecast which has obvious prediction error. In this experiment it is concluded that keeping the ARMA model prediction step length within the period of 35 , it can keep the relative error within $10 \%$, the prediction precision is higher.Acknowledgements

\section{References}

[1]Hu Zewen,Xiao Mingqing.Research of Fault Prediction Based on Time Sequence model[J].

Computer Measurement \&Control,2013,21(6):1421-1423

[2]Sun Bo , Kang Rui , Zhang Shunong.An Approach to Diagnostics and Prognostics Based on Evolutionary Feature Parameters[J].Acta Aeronautica ET Astronautica Sinica,2008,29(2):393-398

[3]Zuo Xianzhang,Kang Jian,Li Hao,Tang Li-wei,Overview of Fault Prediction Technology[J].

Fire Control \& Command Control,2010,35(1):1-5

[4]Lv Chen,Luan Jiahui.Fault diagnosis and prediction: principles, techniques and applications[M].

Beijing:Beihang University Press,2012.12:262-263

[5]Jmes D.Hamilton(original work),Xia Xiaohua(translated),Time Series Analysis[M].Beijing: China Renmin University Press,2015,1,1:49-70

[6]Chen Shuangjin.Time Series Unit Root Test Comparison[D].2013,University of Electronic Science and Technology of China.5-27

[7]An Xiaoxiao,The Model About ARMA and Its Application[D].2008,Yanshan University.33-40 